\title{
Wald Entropy for Ghost-Free, Infinite Derivative Theories of Gravity
}

\author{
Aindriú Conroy, ${ }^{1}$ Anupam Mazumdar, ${ }^{1,2}$ and Ali Teimouri ${ }^{1}$ \\ ${ }^{1}$ Consortium for Fundamental Physics, Lancaster University, Lancaster LA1 4YB, United Kingdom \\ ${ }^{2}$ IPPP, Durham University, Durham DH1 3LE, United Kingdom
}

(Received 1 April 2015; published 21 May 2015)

\begin{abstract}
In this Letter, we demonstrate that the Wald entropy for any spherically symmetric black hole within an infinite derivative theory of gravity that is quadratic in curvature is determined solely by the area law. Thus, the infrared behavior of gravity is captured by the Einstein-Hilbert term, provided that the massless graviton remains the only propagating degree of freedom in the spacetime.
\end{abstract}

DOI: 10.1103/PhysRevLett.114.201101

Einstein's general theory of relativity is a well-behaved theory of gravity in the infrared (IR), reducing to Newtonian predictions in the linearized limit, complete with a slowly varying source term, at large time scales and at large distances. The theory has been tested from solarsystem to cosmological distances [1]. Moreover, gravity has been tested at short distances, and there has been no departure from the $1 / r$ fall of Newtonian potential up to $10^{-5} \mathrm{~m}[2]$.

One of the most intriguing properties of general relativity is that the gravitational entropy of any gravitationally bound system, as in the case of a black hole, follows an area law, depicting gravity as a hologram $[3,4]$. This has been corroborated by the Bekenstein-Hawking entropy of a black hole [5,6], as well as Wald's interpretation of gravitational entropy [7]. The entropy of a black hole has been the cornerstone of many advancements in theoretical physics, for instance, in the context of anti-de Sitter (AdS) and conformal field theory (CFT) correspondence [8].

It is therefore curious to ask a question - what happens to the area law of a gravitational entropy if gravity itself gets modified in the ultraviolet (UV)? Could there be a way to predict the form of higher-order corrections in the gravitational sector from the well-known result of gravity being holographic? In some sense, one may ask-what kind of corrections in the UV in the gravitational sector would one require to maintain the holographic nature of gravity?

Note that the simplicity of general relativity also leads to problems in the ultraviolet (UV). At short distances and at small time scales, the Ricci curvature blows up and, so too, the other observables. The theory admits well-known spacetime singularities, for instance, the black-hole singularity, which is an incarnation of the Newtonian potential; i.e., the potential blows up close to any point source. Irrespective of the mass of the source term, the theory admits a singular solution, known as Schwarzschild's metric, within a static limit. On the other hand, the theory also admits a cosmological singularity appearing at small time scales, which can be seen even in a homogeneous and
PACS numbers: 04.50.Kd, 04.50.-h, 04.60.Cf, 04.70.Dy

an isotropic background solution, such as the FriedmannLemaître-Robertson-Walker metric.

The quantum corrections to the Einstein's gravity may emerge even before the four-dimensional Planck scale, $M_{p}=\sqrt{1 /(8 \pi G)}$. The most general, higher-order action for gravity that is also generally covariant can be written (in four dimensions) as follows:

$$
\begin{aligned}
S^{\mathrm{tot}} & =S^{\mathrm{EH}}+S^{\mathrm{UV}}, \\
S^{\mathrm{EH}} & =\frac{1}{16 \pi G} \int d^{4} x \sqrt{-g} R, \\
S^{\mathrm{UV}} & =\int d^{4} x \sqrt{-g}\left[R^{\mu_{1} \nu_{1} \lambda_{1} \sigma_{1}} \mathcal{O}_{\mu_{1} \nu_{1} \lambda_{1} \sigma_{1}}^{\mu_{2}, \nu_{2} \sigma_{2}} R_{\mu_{2}, \nu_{2} \lambda_{2} \sigma_{2}}\right],
\end{aligned}
$$

where the operator, $\mathcal{O}_{\mu_{1} \nu_{1} \lambda_{1} \sigma_{1}}^{\mu_{2} \nu_{2} \lambda_{2}}$, contains covariant operators such as the D'Alembertian operator $\square=g^{\mu \nu} \nabla_{\mu} \nabla_{\nu}$, where the indices run from $0,1,2,3$. The diffeomorphism could be extended to include contributions that are of higher order in curvature, such as cubic in curvature, quartic in curvature, and so on, but here we shall concentrate on the lowest-order correction, i.e., quadratic in curvature.

Every operator $\square$ comes with a scale, $M$, which could potentially lie anywhere between $(10 \mu \mathrm{m})^{-1} \sim 100 \mathrm{meV} \leq$ $M \leq 10^{19} \mathrm{GeV}$. In the context of string theory, the scale $M$ could be the Kaluza-Klein scale or the compactification scale in four dimensions.

Even if we restrict ourselves to the lowest order, say quadratic in curvature, there are infinitely many covariant derivatives around Minkowski space $[9,10]$. These corrections are expected to arise very naturally in string field theory $[11,12]$, where it is analogous to having all orders of $\alpha^{\prime}$ corrections.

The aim of this Letter is to compute the Wald gravitational entropy for the above infinite higher-derivative action for a static, spherically symmetric background in four dimensions. In particular, this Letter will establish a very intriguing link between the propagating degree of freedom for the graviton and the gravitational entropy. The upshot is as follows: As long as a higher-derivative theory of gravity 
does not introduce any extra propagating degree of freedom, and as long as the IR limit of such a theory yields Einstein-Hilbert (EH) action, the contribution to the Wald entropy due to the higher-derivative corrections must vanish, yielding the famous area law of gravitational entropy, thus preserving the holographic nature of gravity. As a consequence, the gravitational entropy of a black hole for a UV-modified gravity such as in Eq. (1) will still be given by the area law.

We begin by noting that the above action Eq. (1) can be simplified a great deal. The differential operator acting on the right Riemann tensor yields terms which can be integrated by parts. Coupling this with the Bianchi identities and the symmetry properties of the Riemann tensor, it has been shown that the above action can be recast as [9]

$$
\begin{aligned}
S^{\mathrm{tot}}= & \frac{1}{16 \pi G} \int d^{4} x \sqrt{-g}\left\{R+\alpha\left[R \mathcal{F}_{1}\left(\square_{M}\right) R\right.\right. \\
& \left.\left.+R_{\mu \nu} \mathcal{F}_{2}\left(\square_{M}\right) R^{\mu \nu}+R_{\mu \nu \lambda \sigma} \mathcal{F}_{3}\left(\square_{M}\right) R^{\mu \nu \lambda \sigma}\right]\right\},
\end{aligned}
$$

where $\alpha$ has inverse of mass squared dimension, and we have defined $\square_{M} \equiv \square / M^{2}$ for convenience. The $\mathcal{F}$ 's are the three unknown analytic functions given by

$$
\mathcal{F}_{i}\left(\square_{M}\right)=\sum_{n=0}^{\infty} f_{i_{n}}\left(\square_{M}\right)^{n},
$$

where $f_{i_{n}}$ are appropriate constants. The question we are keen to explore is as follows: Is there any deep connection between the $\mathcal{F}$ 's and the gravitational entropy? In order to address this, let us now consider a simple static, homogeneous, and isotropic metric of the type

$$
d s^{2}=-f(r) d t^{2}+f(r)^{-1} d r^{2}+r^{2} d \Omega^{2},
$$

where $\Omega$ denotes the angular coordinates. This metric has asymptotic behavior in all three cases, i.e., Minkowski, de Sitter, and anti-de Sitter. The gravitational entropy for any such metric can be defined in terms of the Wald entropy [7], see also [13]. The definition of Wald's entropy follows the Bekenstein-Hawking area law of a black hole and the first law of black-hole thermodynamics, which has a clear geometric interpretation through its identification with the Noether charge for spacetime diffeomorphisms. Indeed, Wald explains that by varying Lagrangian density with respect to all fields, including the metric, and by using the diffeomorphism invariance of the theory, one can obtain the associated Noether current, and, hence, the Noether charge. Hence, the gravitational entropy can be recast as a closed integral over a cross section of the horizon for the metric given by Eq. (4).

For a spherically symmetric black-hole solution in four dimensions, the Wald entropy can be written as [7]

$$
S_{W}=-2 \pi \oint\left(\frac{\delta \mathcal{L}}{\delta R_{a b c d}}\right)^{(0)} \hat{\epsilon}_{a b} \hat{\epsilon}_{c d} q(r) d \Omega^{2},
$$

where $\mathcal{L}$ is the Lagrangian, $\hat{\epsilon}_{a b}$ is the binormal vector to the surface, where the indices $\{a, b, c, d\} \in\{r, t\}$, and $q(r) d \Omega^{2}=r^{2}\left(d \theta^{2}+\sin ^{2} \theta d \phi^{2}\right)$. The superscript “( $(0)$ " indicates that the functional derivative is determined on the background and the factor of 4 arising due to the antisymmetric properties of the Riemann tensor and the binormal vectors. We can then write the Wald entropy as

$$
S_{W}=-8 \pi \oint\left(\frac{\delta \mathcal{L}}{\delta R_{r t r t}}\right)^{(0)} q(r) d \Omega^{2} .
$$

In general, one can construct two normal directions along $r$ and $t$ with $\oint \equiv \oint_{r=r_{H}, t=\mathrm{const}}$. Moreover, the area of the horizon is defined to be

$$
\text { Area }=\oint q(r) d \Omega^{2}
$$

The Wald entropy corresponding to Eq. (2) can be computed by calculating the functional derivatives of every term in Eq. (2), resulting in two distinct contributions to the entropy,

$$
S_{W}=S_{W}^{\mathrm{EH}}+S_{W}^{\mathrm{UV}},
$$

given by

$$
\begin{aligned}
S_{W}= & \frac{1}{4 G} \oint\left[1+\alpha\left\{2 \mathcal{F}_{1}\left(\square_{M}\right) R\right.\right. \\
& -\mathcal{F}_{2}\left(\square_{M}\right) \times\left(g^{r r} R^{t t}+g^{t t} R^{r r}\right) \\
& \left.\left.-4 \mathcal{F}_{3}\left(\square_{M}\right) R^{r t r t}\right\}\right] q(r) d \Omega^{2} .
\end{aligned}
$$

For a spherically symmetric metric of the type given by Eq. (4), one can read off the following identities: $g_{t t} g_{r r}=-1, \quad g_{t t}=-g^{r r}, \quad g_{r r}=-g^{t t} . \quad$ Subsequently, $\left(g^{r r} R^{t t}+g^{t t} R^{r r}\right)=-g_{a b} R^{a b}=-R$, and similarly $-2 R^{r t r t}=$ $2 g_{t t} g_{r r} R^{r t r t}=g^{a b} g^{c d} R_{d a c b}=R$. With the help of these identities, we can further simplify the above expression,

$S_{W}=\frac{\text { Area }}{4 G}\left[1+\alpha\left\{2 \mathcal{F}_{1}\left(\square_{M}\right)+\mathcal{F}_{2}\left(\square_{M}\right)+2 \mathcal{F}_{3}\left(\square_{M}\right)\right\} R\right]$.

Interestingly, at large distances from any source term, such as in the case of IR, the action Eq. (2) is dominated by the Einstein-Hilbert term. It is a well-known result that for the Einstein-Hilbert action, the Wald entropy is given by $S_{W}=$ Area/4G. The UV part of the gravitational entropy contains a very interesting combination of $\mathcal{F}$ 's, which will play a crucial role in understanding the UV aspects of gravity and its entropy. The profound question arises - could we constrain the nature of the $\mathcal{F}$ 's to some 
fundamental aspects of how gravity should be modified in the UV?

Let us also note that this modified action, Eq. (2), will inevitably modify the graviton propagator. If the $\mathcal{F}$ 's contain infinite derivatives, it would inevitably modify the graviton propagator in the UV. It is well known that higher derivative theories have ghosts at tree level; for instance, the fourth derivative gravity of Stelle [14] contains a massive ghost. Therefore, it is paramount to understand the nature of the graviton propagator and its connection to Wald's entropy.

The exact form of the propagator for the above action, Eq. (2), was derived in Refs. $[9,15]$. In principle the propagator can be recast in terms of the spin projection operators [16], such as the tensor $P^{2}$ and the scalar operator $P_{s}^{0}$, in the momentum space $[9,15]$

$$
\Pi\left(k^{2}\right) \sim \frac{P^{2}}{a\left(k^{2}\right) k^{2}}+\frac{P_{s}^{0}}{\left[a\left(k^{2}\right)-3 c\left(k^{2}\right)\right] k^{2}},
$$

where $a\left(k^{2}\right), c\left(k^{2}\right)$ can be written in terms of the original $\mathcal{F}$ 's contained within the modified action, as $[9,15]$

$$
\begin{aligned}
& a\left(\square_{M}\right)=1-\frac{1}{2} \mathcal{F}_{2}\left(\square_{M}\right) \square_{M}-2 \mathcal{F}_{3}\left(\square_{M}\right) \square_{M}, \\
& c\left(\square_{M}\right)=1+2 \mathcal{F}_{1}\left(\square_{M}\right) \square_{M}+\frac{1}{2} \mathcal{F}_{2}\left(\square_{M}\right) \square_{M} .
\end{aligned}
$$

Further, since we wish to recover general relativity in the IR, i.e., $\square \rightarrow 0, k^{2} \rightarrow 0$, we must have

$$
a(0)=c(0)=1,
$$

corresponding to the massless graviton propagator for the Einstein-Hilbert action. Now assuming that $a\left(\square_{M}\right)=$ $c\left(\square_{M}\right)$, such that we can take a continuous limit from $\mathrm{UV} \rightarrow \mathrm{IR}$, we have

$$
\lim _{k^{2} \rightarrow 0} \Pi \sim \frac{1}{a\left(k^{2}\right)}\left[\frac{P^{2}}{k^{2}}-\frac{P_{s}^{0}}{2 k^{2}}\right] \rightarrow\left[\frac{P^{2}}{k^{2}}-\frac{P_{s}^{0}}{2 k^{2}}\right] .
$$

From the above Eqs. (11)-(15), there are some crucial observations to make.

Ghost-free condition.- -Note that UV modifications of gravity should be such that the action must have a smooth IR limit. It follows that Eqs. (14), (15) must be satisfied; i.e., the $k^{2}=0$ pole just describes the physical graviton state. This also implies that the action Eq. (2) maintains causality and the ghost-free condition as long as there is no new pole introduced by the analytic function $a\left(\square_{M}\right)$. The fact that the theory must be ghost free boils down to simply requiring that $a\left(\square_{M}\right)$ is an entire function; because $a\left(\square_{M}\right)-3 c\left(\square_{M}\right)$ has at most a single zero, the corresponding residue at the pole would necessarily have the correct sign, since the entire function does not have any poles in the complex plane, containing only an essential singularity at the boundary [9].

Essentially, the above Eq. (14) means that $a\left(\square_{M}\right)$ and $c\left(\square_{M}\right)$ are nonsingular analytic functions at $k^{2}=0$ and, therefore, cannot contain nonlocal inverse derivative operators.

Constraints.-Because we do not wish to introduce any new extra degrees of freedom other than the massless graviton throughout the IR to the UV, we require a constraint relationship between the $\mathcal{F}$ 's from Eqs. (12), (13),

$$
\begin{aligned}
a\left(\square_{M}\right)= & c\left(\square_{M}\right) \Rightarrow 2 \mathcal{F}_{1}\left(\square_{M}\right)+\mathcal{F}_{2}\left(\square_{M}\right) \\
& +2 \mathcal{F}_{3}\left(\square_{M}\right)=0 .
\end{aligned}
$$

The above conclusions have intriguing consequences for the gravitational entropy-reducing Eq. (16) to the Wald entropy, see Eq. (10), of a spherically symmetric black hole, as is the case for the standard result of $S^{\mathrm{EH}}$,

$$
S_{W}=S_{W}^{\mathrm{EH}}=\frac{\text { Area }}{4 G} .
$$

This is an important result: The holographic nature of gravity remains preserved in spite of the nontrivial modifications of gravity with infinite derivatives in the UV. The UV contribution to the gravitational entropy is simply $S_{W}^{\mathrm{UV}}=0$, for a metric given by Eq. (4). This result is a sheer consequence of the graviton being massless, the requirement of not introducing of any new propagating degrees of freedom for the graviton, and is independent of the choice of $\mathcal{F}_{i}$ 's in the action Eq. (2).

This leaves us with a profound question: Why is the gravitational entropy $S_{W}^{\mathrm{UV}}=0$ ? As such, our constraint Eq. (16) is very generic and, other than the massless nature of the graviton, does not shed any light on the nature of gravity in the UV. A priori, we do not know whether the gravitational interaction in the UV becomes weak or strong. However, the form of the $\mathcal{F}$ 's does tell us of some interesting aspects of gravity in the UV-namely, the gravitational interaction becomes nonlocal $[9,10]$ and helps us to understand the quantum behavior of gravity at higher loops; i.e., above one loop there are indications that the theory is convergent [17-20], and explicit computations have been performed in a toy model up to two loops [20]. Further note that $S_{W}^{\mathrm{UV}}=0$ will have a very interesting consequence for the third law of thermodynamics in the context of gravity, which might hint towards the absolute ground state of gravity when the condition Eq. (16) is imposed for the action $S^{\mathrm{UV}}$.

Gravity, being a gauge theory, contains all its interactions within the kinetic term. If the graviton propagator is modified by Eq. (15), the vertex factor for any gravitongraviton interaction will also be enhanced by a factor $a\left(k^{2}\right)$. One such study has been performed in the context of singularity-free gravity [20], where the form of $a\left(k^{2}\right) \sim$ $e^{k^{2} / M^{2}}$ has been suggested, motivated by string field theory, 
where the vertex operator gets similarly exponentially enhanced. In this particular case, it has been shown that the black-hole singularity for a spherically symmetric metric disappears in the linearized limit, therefore ameliorating the UV nature of gravity [9]. (Note that the full nonlinear equations of motion are extremely difficult to tackle, see [21]. So far only a cosmological solution has been constructed from the full equations of motion [10].)

At this point, however, it seems Eq. (17) is a very generic prediction for such an infinite derivative theory of gravity, irrespective of the actual form of $a\left(\square_{M}\right)$, as long as $a\left(\square_{M}\right)$ does not contain any additional poles.

So far, our analysis is very generic and applicable to the full action Eq. (2). We may gain further insight into the Newtonian potentials of the metric by assessing the linearized metric, such that it becomes an asymptotically flat spacetime. Let us assume that the $(t, r)$ component of the original spherically symmetric metric, Eq. (4), takes the form

$$
d s^{2}=-[1+2 \Phi(r)] d t^{2}+[1-2 \Psi(r)] d r^{2},
$$

where $2 \Phi(r), 2 \Psi(r) \ll 1$. In fact, $\Phi$ and $\Psi$ are the two Newtonian potentials. One can then ask: What should be the Wald entropy in the linearized limit of the action given by Eq. (2)? For the above metric, Eq. (18), we can evaluate the Wald entropy, simplifying the expression by considering a static solution. The gravitational entropy is then given, at the linearized order, by

$$
\begin{aligned}
S_{W}= & \frac{\text { Area }}{4 G}\left\{1+2 \Psi-2 \Phi+\alpha\left[2 \mathcal{F}_{1}\left(\square_{M}\right)+\mathcal{F}_{2}\left(\square_{M}\right)\right.\right. \\
& \left.\left.+2 \mathcal{F}_{3}\left(\square_{M}\right)\right]\left(-2 \Phi^{\prime \prime}\right)\right\},
\end{aligned}
$$

where ' denotes the derivative with respect to $r$. Note that when $\Psi=\Phi$ and $2 \mathcal{F}_{1}+\mathcal{F}_{2}+2 \mathcal{F}_{3}=0$, for any source term within the linearized limit, the gravitational entropy duly reduces to that of the $S_{W}^{\mathrm{EH}}$. The conditions are exactly the same as that of our complete analysis. Indeed, it would be interesting to seek scenarios when the area law of a black hole might incur modifications. One might imagine departing from the assumption of spherical symmetry, in which case it is possible to realize $\Phi \neq \Psi$.

However, the other possibility, when $a\left(\square_{M}\right) \neq c\left(\square_{M}\right)$, is more interesting. This condition would immediately imply that there are additional poles in the graviton propagator other than the massless graviton, see Eqs. (11), (15). For instance, $\mathcal{L} \sim f(R)$ gravity, which is very popular due to its simplicity, contains an extra scalar degree of freedom other than the massless graviton, see [15]. Any UV modification with $f(R)$ gravity would therefore contribute to the gravitational entropy in addition to $S_{W}^{\mathrm{EH}}$. However, such class of gravity does not ameliorate the UV aspects of gravity at all [9]. Similarly, the conformal invariant gravity, $\mathcal{L}=R-\alpha C^{2}$, contains a massive spin-2 degree of freedom other than the massless graviton $[9,15]$. Moreover, this massive spin-2 degree of freedom comes with a wrong sign in the graviton propagator, thus revealing a massive ghost. Both of these examples are a subset of the above action Eq. (2), and suggest that one of the $\mathcal{F}$ 's should be zero. Of course, a consequence of such a vanishing function $\mathcal{F}$ is that the action would be incomplete from the UV point of view.

Before we conclude, let us briefly bring the reader's attention to this final intriguing point. The condition $a\left(\square_{M}\right) \neq c\left(\square_{M}\right)$ seems to have some relevance for cosmology. Unlike the black-hole case, the cosmological singularity cannot be avoided by assuming $a\left(\square_{M}\right)=$ $c\left(\square_{M}\right)$ as shown in $[10,22,23]$. One requires additional degrees of freedom other than the massless graviton, which remains a tantalizing issue- this leads one to ask why the respective natures of these two singularities are so different, and why the fundamental nature of the graviton has to deviate to understand these two problems.

In conclusion, we have found a very intriguing result for a class of ghost-free, infinite derivative theories of gravity - the gravitational entropy for a spherically symmetric metric is solely given by the Einstein-Hilbert action. The area law of gravitational entropy is the main contribution arising from the IR aspect of gravity, while the UV contribution (from an action up to quadratic in curvature) of the gravitational entropy vanishes exactly. This happens due to an interesting connection between the propagating degrees of freedom for the graviton: If the massless graviton remains the only propagating degree of freedom in the spacetime, then there will be no other contribution to the gravitational entropy other than the Einstein-Hilbert term's contribution. In generality, at least in the spherically symmetric case, gravity remains holographic. Our result, $S_{W}^{\mathrm{UV}}=0$, will have some profound consequences for the third law of thermodynamics in the gravitational system, which we shall explore in the future.

A. C. is funded by STFC Grant No. ST/K50208X/1, and A. M. is supported by STFC Grant No. ST/J000418/1. A. M. acknowledges the kind hospitality from IPPP, Durham, during the course of this work.

[1] C. M. Will, Living Rev. Relativity, 17, 4 (2014).

[2] E. G. Adelberger, B. R. Heckel, S. A. Hoedl, C. D. Hoyle, D. J. Kapner, and A. Upadhye, Phys. Rev. Lett. 98, 131104 (2007).

[3] G. 't Hooft, in Salamfestschrift: A Collection of Talks from the Conference on Highlights of Particle and Condensed Matter Physics ICTP, Trieste, Italy, 8-12 March 1993, World Scientific Series in 20th Century Physics Vol. 4 (World Scientific, Singapore, 1993), pp. 284-296.

[4] L. Susskind, J. Math. Phys. (N.Y.) 36, 6377 (1995).

[5] J. D. Bekenstein, Phys. Rev. D 7, 2333 (1973); Lett. Nuovo Cimento 4, 737 (1972). 
[6] S. W. Hawking, Nature (London) 248, 30 (1974); Commun. Math. Phys. 43, 199 (1975).

[7] R. M. Wald, Phys. Rev. D 48, R3427(R) (1993); V. Iyer and R. M. Wald, Phys. Rev. D 50, 846 (1994).

[8] J. M. Maldacena, Int. J. Theor. Phys. 38, 1113 (1999); Adv. Theor. Math. Phys. 2, 231 (1998).

[9] T. Biswas, E. Gerwick, T. Koivisto, and A. Mazumdar, Phys. Rev. Lett. 108, 031101 (2012).

[10] T. Biswas, A. Mazumdar, and W. Siegel, J. Cosmol. Astropart. Phys. 03 (2006) 009.

[11] E. Witten, Nucl. Phys. B268, 253 (1986).

[12] W. Siegel, arXiv:hep-th/0107094.

[13] T. Jacobson, G. Kang, and R. C. Myers, Phys. Rev. D 49, 6587 (1994).

[14] K. S. Stelle, Phys. Rev. D 16, 953 (1977).

[15] T. Biswas, T. Koivisto, and A. Mazumdar, arXiv:1302.0532.

[16] P. Van Nieuwenhuizen, Nucl. Phys. B60, 478 (1973).
[17] E. Tomboulis, Phys. Lett. 97B, 77 (1980); E. T. Tomboulis, arXiv:hep-th/9702146.

[18] W. Siegel, arXiv:hep-th/0309093.

[19] L. Modesto, Phys. Rev. D 86, 044005 (2012).

[20] S. Talaganis, T. Biswas, and A. Mazumdar, arXiv:1412.3467.

[21] T. Biswas, A. Conroy, A. S. Koshelev, and A. Mazumdar, Class. Quantum Grav. 31, 015022 (2014); 31, 159501(E) (2014); H. J. Schmidt, Class. Quantum Grav. 7, 1023 (1990).

[22] T. Biswas, T. Koivisto, and A. Mazumdar, J. Cosmol. Astropart. Phys. 11 (2010) 008; T. Biswas, A. S. Koshelev, A. Mazumdar, and S. Y. Vernov, J. Cosmol. Astropart. Phys. 08 (2012) 024; A. Conroy, A. S. Koshelev, and A. Mazumdar, Phys. Rev. D 90, 123525 (2014).

[23] A. S. Koshelev and S. Y. Vernov, Phys. Part. Nucl. 43, 666 (2012); G. Calcagni, L. Modesto, and P. Nicolini, Eur. Phys. J. C 74, 2999 (2014). 